\title{
Charges of COVID-19 Diagnostic Testing and Antibody Testing Across Facility Types and States
}

$\mathrm{J}$ Gen Intern Med

DOI: $10.1007 /$ s11606-020-06198-y

(c) Society of General Internal Medicine 2020

\section{INTRODUCTION}

The high charges for COVID-19 testing by some healthcare providers have received broad media and public attention. ${ }^{1}$ Individual providers determine their own charge, which does not vary with insurance type. ${ }^{2,3}$ The charges for COVID-19 testing have important implications for out-of-network plans, uninsured patients, and other payers with little negotiating power. The CARES Act requires that private plans that do not have a negotiated rate with the provider pay the price publicly listed by the provider for COVID-19 testing, which is usually the same as or a percentage of the charge. ${ }^{4,5}$ Providers that have tested uninsured patients can choose to either seek reimbursement from the Department of Health and Human Services or bill uninsured patients at a self-determined price, which equals the charge unless the provider offers a discount. Therefore, the higher the charge for COVID19 testing, the higher the potential cost exposure of plans without negotiating power and of uninsured patients. Nationwide COVID-19 testing charges across facility types remain unexplored. In this study, we aim to examine the charges for the most commonly performed COVID-19 diagnostic test (CPT code: 87635) and antibody test (CPT code: 86769 ) across facility types and states.

\section{METHODS}

We obtained administrative claims data for COVID-19 testing from the COVID-19 Research Database, a pro bono cross-industry collaborative. ${ }^{6}$ The sample consisted of 182,149 claims of diagnostic testing (CPT code: 87635, Medicare rate \$51.31) from 2324 providers in 50 states and DC, and 318,546 claims of antibody testing (CPT code: 86769, Medicare rate \$42.13) from 764 providers in 46 states and DC. All claims were submitted between March 19 and July 19, 2020. We obtained the state-level diagnostic testing rate, infection

Received August 1, 2020

Accepted August 27, 2020 rate, and mortality rate, as of July 20, 2020, from the Johns Hopkins Coronavirus Resource Center.

For each test and facility type, we calculated the average charge (total charges divided by total claims and compared across facility types). For each test and state (with more than ten claims), we calculated the average charge and grouped the states into low, medium, and high tertiles. We analyzed the correlation between each state's average testing charge and its COVID-19 testing rate (available only for diagnostic testing), infection rate, and mortality rate to understand whether the testing charges were influenced by the demand for the test.

\section{RESULTS}

For COVID-19 diagnostic testing, the mean, median, and standard deviations of charges were $\$ 144.06, \$ 100.00$ (IQR \$67.00-\$155.00), and \$162.18. The most common facility type was independent laboratories (performed $49.7 \%$ of all tests), with an average charge of $\$ 140.41$ (range \$0.01-\$14,750.00, IQR \$67.00-\$100.00), followed by hospital outpatient settings (performed $34.5 \%$ of all tests), with an average charge of $\$ 168.87$ (range \$0.01-\$2436.00, IQR \$94.00-\$204.00) (Fig. 1, panel a). For antibody testing, the mean, median, and standard deviations of charges were \$63.93, \$55.00 (IQR \$42.13-\$55.00), and \$48.92. Independent laboratories performed $97.2 \%$ of all tests, with an average charge of $\$ 62.30$ (range \$4.37-\$1515.00; IQR \$42.13-\$55.00) (Fig. 1, panel b). In total, $8.0 \%$ of diagnostic testing services and $14.0 \%$ of antibody testing claims were charged one standard deviation above the mean ( $\$ 306.24$ for diagnostic testing and $\$ 112.85$ for antibody testing).

The state average testing charges ranged between $\$ 64.98$ (UT) and $\$ 505.65$ (DC) for diagnostic testing, and $\$ 45.85(\mathrm{NY})$ and $\$ 195.41(\mathrm{NM})$ for antibody testing. AR, LA, MO, and NM had high average charges for both tests (diagnostic test $>\$ 144.56$; antibody test $>\$ 80.61)$. GA, KS, MA, MD, NC, NV, and OK had low charges for both tests (diagnostic test $\leq \$ 103.15$; antibody test $\leq \$ 56.10$ ) (Fig. 2). No statistically significant association $(P<0.05)$ was found between testing charges and state-level testing rates, infection rates, or mortality rates. 


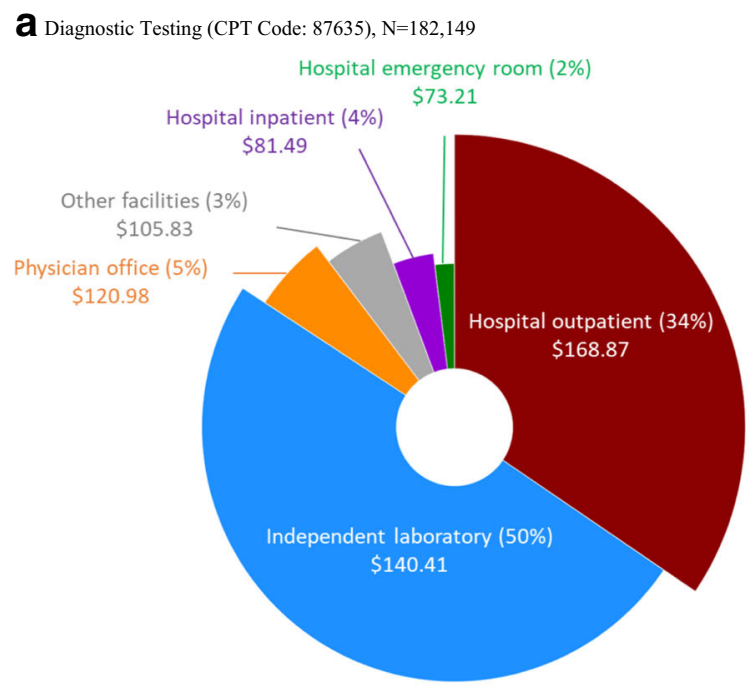

b Antibody Testing (CPT Code:86769), N=318,546

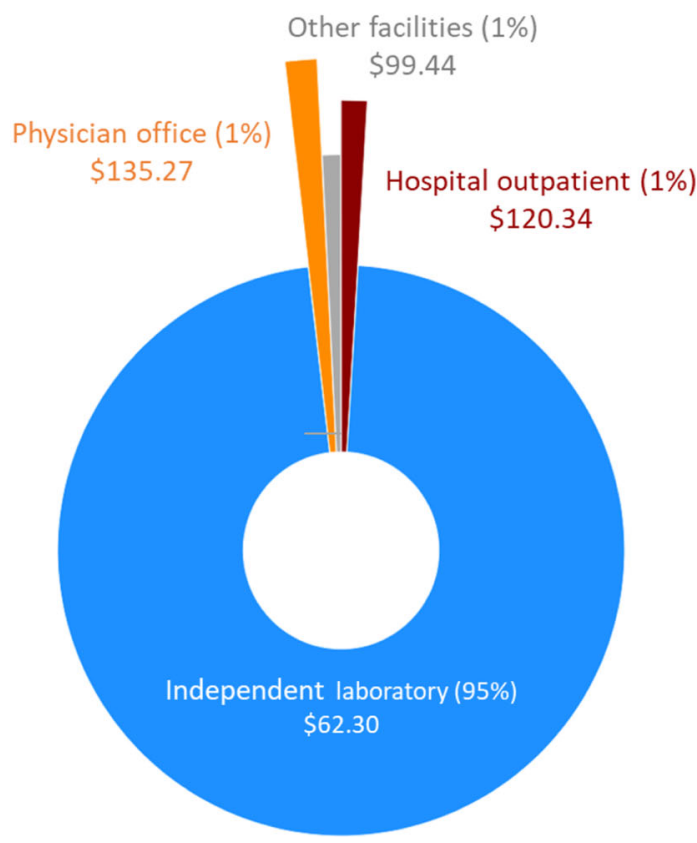

Figure 1 Average charges for COVID-19 testing, by facility type. a Diagnostic testing (CPT code: 87635), $N=182,149$. Other facilities include ambulatory surgical center, ESRD treatment center, federally qualified health center, intermediate care facility, mobile unit, public health clinic, rural health clinic, skilled nursing facility, telehealth, tribal free-standing clinic, urgent care facility, walk-in retail health clinic, and unspecified settings. The width (degrees) of each slice represents the relative service volume. The data include 411 physician offices, 124 hospital inpatient settings, 64 hospital emergency rooms, 1212 hospital outpatient settings, 226 independent laboratories, and 278 other facilities. The radius of each slice represents the average charge. The Medicare reimbursement rate is $\$ 51.31$. b Antibody testing (CPT code: 86769$)$, $N=$ 318,546. Other facilities include ambulatory surgical center, ESRD treatment center, federally qualified health center, public health clinic, rural health clinic, skilled nursing facility, telehealth, tribal free-standing clinic, urgent care facility, walk-in retail health clinic, hospital emergency room, and unspecified settings. The data include 165 independent laboratories, 325 hospital outpatient settings, 214 physician offices, and 60 other facilities. The width (degrees) of each slice represents the relative service volume. The radius of each slice represents the average charge.

The Medicare reimbursement rate is $\$ \mathbf{4 2 . 1 3}$.

\section{DISCUSSION}

A small proportion of COVID-19 diagnostic testing and antibody testing services, provided in selected states, had charges that far exceeded the Medicare rate. High charges may create financial barriers for some uninsured patients and lead to higher premiums for out-of-network private plans. The results, based on a proportion of all testing claims in the USA, might underestimate the national variation of COVID19 testing charges. The insurance and network status of each claim and the actual prices paid were unavailable. 
a Diagnostic Testing (CPT Code: 87635$), \mathrm{N}=182,149$

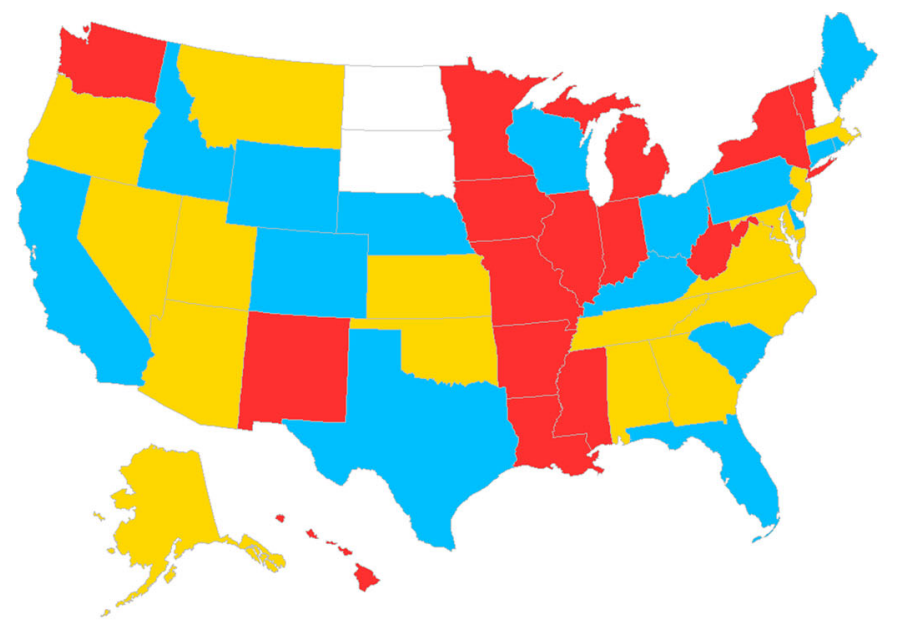

b Antibody Testing (CPT Code: 86769), N=318,546

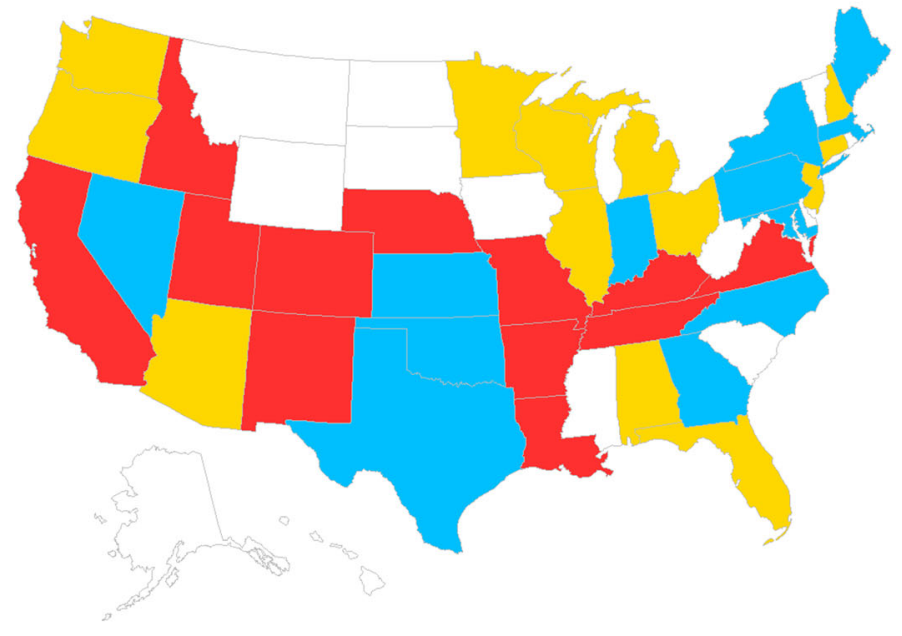

Figure 2 Average charges for COVID-19 testing, by state. a Diagnostic testing (CPT code: 87635), $N=182,149$. States that had ten or fewer claims were classified as "No data." The Medicare reimbursement rate is $\$ 51.31$. b Antibody testing (CPT code: 86769$), N=318,546$. States that had ten or fewer services were classified as "No data." The Medicare reimbursement rate is \$42.13.

Mark K. Meiselbach, $B S c^{1}$

Ge Bai, PhD, CPA ${ }^{1,3}$

Gerard F. Anderson, $\mathrm{PhD}^{1,2}$

${ }^{1}$ Johns Hopkins Bloomberg School of Public Health, Baltimore, MD, USA

${ }^{2}$ Johns Hopkins School of Medicine,

Baltimore, MD, USA

${ }^{3}$ Johns Hopkins Carey Business School,

Baltimore, MD, USA

Corresponding Author: Ge Bai, PhD, CPA; Johns Hopkins Carey Business School, Baltimore, MD, USA (e-mail: gbai@jhu.edu).

\section{Compliance with Ethical Standards:}

Conflict of Interest: All authors declare no potential conflicts of interest with respect to the research, authorship, or publication of this article. Mark K. Meiselbach is supported by the T32HS0OOO29 grant from the Agency for Healthcare Research and Quality. The content is solely the responsibility of the authors and does not necessarily represent the official views of the Agency for Healthcare Research and Quality. The data, technology, and services used in the generation of these research findings were generously supplied pro bono by the COVID-19 Research Database partners, who are acknowledged at www.covid19researchdatabase.org. This study is funded by Arnold Ventures.

\section{REFERENCES}

1. Kliff S. Two friends in Texas were tested for coronavirus. One bill was \$199. The other? \$6,408. New York Times. https://www.nytimes.com/ 2020/06/29/upshot/coronavirus-tests-unpredictable-prices.html. Accessed Jul 28, 2020.

2. Bai G, Anderson GF. Variation in the ratio of physician charges to Medicare payments by specialty and region. JAMA. 2017;317(3):315-8. https://doi.org/10.1001/jama.2016.16230 
3. Bai G, Anderson GF. Extreme markup: the fifty US hospitals with the highest charge-to-cost ratios. Health Aff (Millwood). 2015;34(6):922-928. https://doi.org/10.1377/hlthaff.2014.1414

4. United States Congress. H.R.748 - CARES Act. Accessed at www.congress. gov/bill/116th-congress/house-bill/748 on 28 July 2020.

5. Department of Labor. FAGs about Families First Coronavirus Response Act and Coronavirus Aid, Relief, and Economic Security Act implementation. Accessed at https://www.dol.gov/sites/dolgov/files/ebsa/about-ebsa/ our-activities/resource-center/faqs/aca-part-42.pdf. Accessed Jul 28, 2020.

6. COVID-19 Research Database. https://www.covid19researchdatabase. org. Accessed Jul 28, 2020.

Publisher's Note Springer Nature remains neutral with regard to jurisdictional claims in published maps and institutional affiliations. 